\title{
Secular evolution and pseudo-bulges
}

\author{
Francoise Combes \\ Observatoire de Paris, LERMA, Collège de France, CNRS, \\ PSL University, Sorbonne University, UPMC, Paris \\ email: francoise.combes@obspm.fr
}

\begin{abstract}
Through vertical resonances, bars can produce pseudo-bulges, within secular evolution. Bulges and pseudo-bulges have doubled their mass since $z=1$. The frequency of bulge-less galaxies at $\mathrm{z}=0$ is difficult to explain, especially since clumpy galaxies at high $\mathrm{z}$ should create classical bulges in all galaxies. This issue is solved in modified gravity models. Bars and spirals in a galaxy disk, produce gravity torques that drive the gas to the center and fuel central star formation and nuclear activity. At $0.1-1 \mathrm{kpc}$ scale, observations of gravity torques show that only about one third of Seyfert galaxies experience molecular inflow and central fueling, while in most cases the gas is stalled in resonant rings. At 10-20pc scale, some galaxies have clearly revealed AGN fueling due to nuclear trailing spirals, influenced by the black hole potential. Thanks to ALMA, and angular resolution of up to $80 \mathrm{mas}$ it is possible to reach the central black hole (BH) zone of influence, discover molecular tori, circum-nuclear disks misaligned with the galaxy, and the $\mathrm{BH}$ mass can be derived more directly from the kinematics.
\end{abstract}

Keywords. galaxies: active, galaxies: bulges, galaxies: evolution, galaxies: ISM, galaxies: kinematics and dynamics, galaxies: nuclei, galaxies: Seyfert, galaxies: spiral

\section{Introduction}

The formation of spheroïdal components has been widely studied through numerical simulations and observational surveys, and it is possible to distinguish several scenarios, including secular evolution. There is however a severe problem still open in the high frequency of bulgeless galaxies at $\mathrm{z}=0$, which is difficult to account for in the standard model. Secular evolution through bars is able to form box-peanut shape bulges, and some are now observed also in nuclear bars. Observations are also able to study the evolution of pseudo-bulges with redshift.

Bars and embedded structures (nuclear bars and spirals) are driving gas towards the center of galaxies through gravity torques and fuel the central black hole. With the recent progress in angular resolution and sensitivity, we can follow this phenomenon down to the 10pc scales. It is now possible to detect the molecular tori around nearby Seyfert nuclei, and to disentangle fueling and molecular outflows.

\section{The problem of bulgeless galaxies}

Classical bulges and spheroïds of early-type galaxies are mostly formed in mergers, either a small number of major mergers, or a large number of minor ones, both cancelling out the resulting angular momentum. Pseudo-bulges are distinct from classical ones, by being more similar to disks, through their flattening, their rotation level, and their light profile (Sersic of index $\mathrm{n}<3$ ). They are mostly the results of internal processes, namely vertical resonance with a bar, able to elevate stars in the center into a thick and spheroïdal component, more precisely with a peanut-shape corresponding to the resonance radius.

Galaxies at high redshift are observed clumpy, with 4-5 clumps of at least $10^{9} \mathrm{M}_{\odot}$, due 

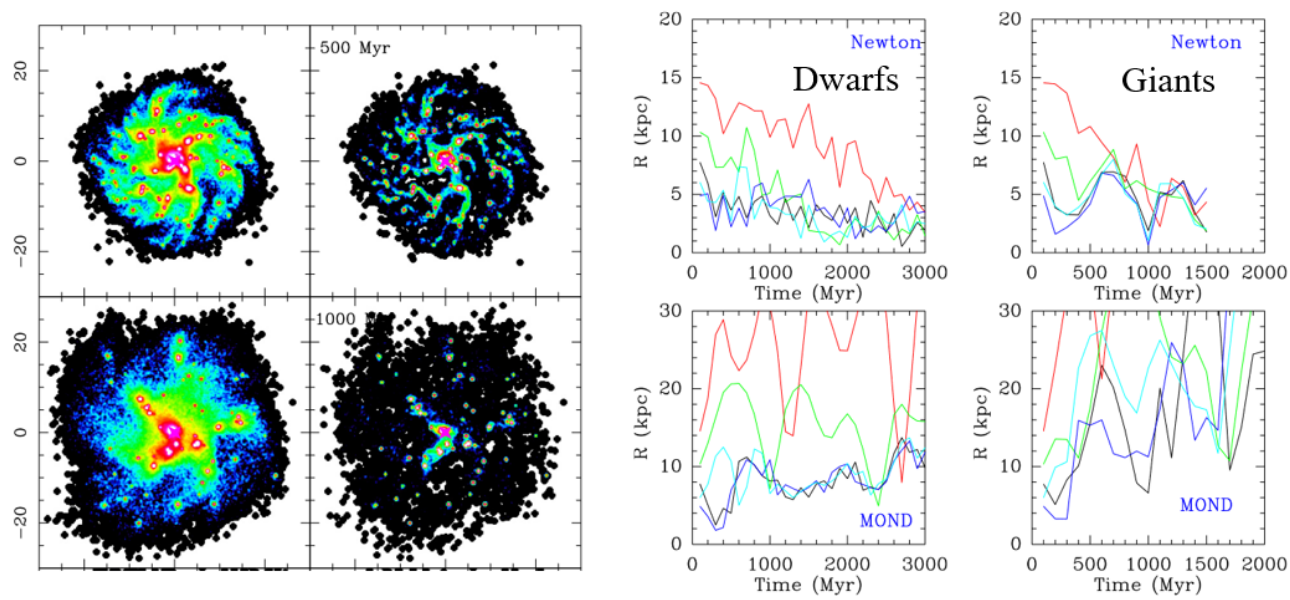

Figure 1. The left panels (each $60 \mathrm{kpc}$ in size) correspond to the MOND simulation of a dwarf galaxy, imaging all baryons (left) and gas (right) surface densities, at epochs 0.5, and 1 Gyr. The right panels show the radial decay of the 5 main clumps, in the Newtonian+DM (top) and MOND (bottom) for the dwarf (left) and giant (rignt) galaxies (Combes 2014).

to their high gas content and consequent instability. These clumps can also form a bulge, through dynamical friction on the dark matter halo. This makes the problem of the high frequency of bulgeless galaxies today more acute (Kormendy \& Fisher 2008 , Weinzirl et al. 2009). Simulations in the standard model have shown that the supernovae feedback is not rapid enough to destroy the clumps before their spiraling to the center (Ceverino et al. 2010).

It is however possible to reduce considerably the dynamical friction, in a modified gravity model, where there is no dark matter particules, only friction on the disk itself. Simulations of gaseous galaxies, both dwarfs and giants, have been carried out with the same star-forming and feedback recipes in MOND and Newton+DM models (Combes 2014). The clumpy galaxies can evolve into bulgeless galaxies today (cf Figure 1). In this model, there are also much less galaxy mergers, so less massive spheroïds.

\section{Pseudo-bulges: peanut in nuclear bars, ans evolution with $\mathrm{z}$}

The frequency of pseudo-bulges has been shown to depend essentially on stellar mass (Fisher \& Drory 2010). They dominate at stellar masses lower than $310^{10} \mathrm{M}_{\odot}$, while classical bulges dominate at larger masses. Their frequency depends also on environment: there is half less pseudo-bulges in centrals with respect to satellites and field galaxies (Mishra et al. 2017). Pseudo-bulges and pure disks are more frequent in sheets, and classical bulges in filaments and halos (Wang et al. 2019). Small mass galaxies with classical bulges have more local neighbors. For them, intermediate to large-scale environment is not important: They are associated to neighbors, and are likely due to interactions and mergers.

The growth of bulges in galaxies since $\mathrm{z}=1$ has been studied by Sachdeva et al. (2017). The mass in pseudo-bulges is about half of the mass of the classical ones. Both bulges double in mass since $\mathrm{z} \sim 1$ : the bulge to total stellar mass ratio increases from 10 to $26 \%$ for pseudo-bulge galaxies, and from 21 to $52 \%$ for classical ones. Across redshifts, the growth of bulges is accompanied by the fading of their disk, which could be attributed to secular evolution (Sachdeva et al. 2017). Pseudo-bulges are in general less bright than 

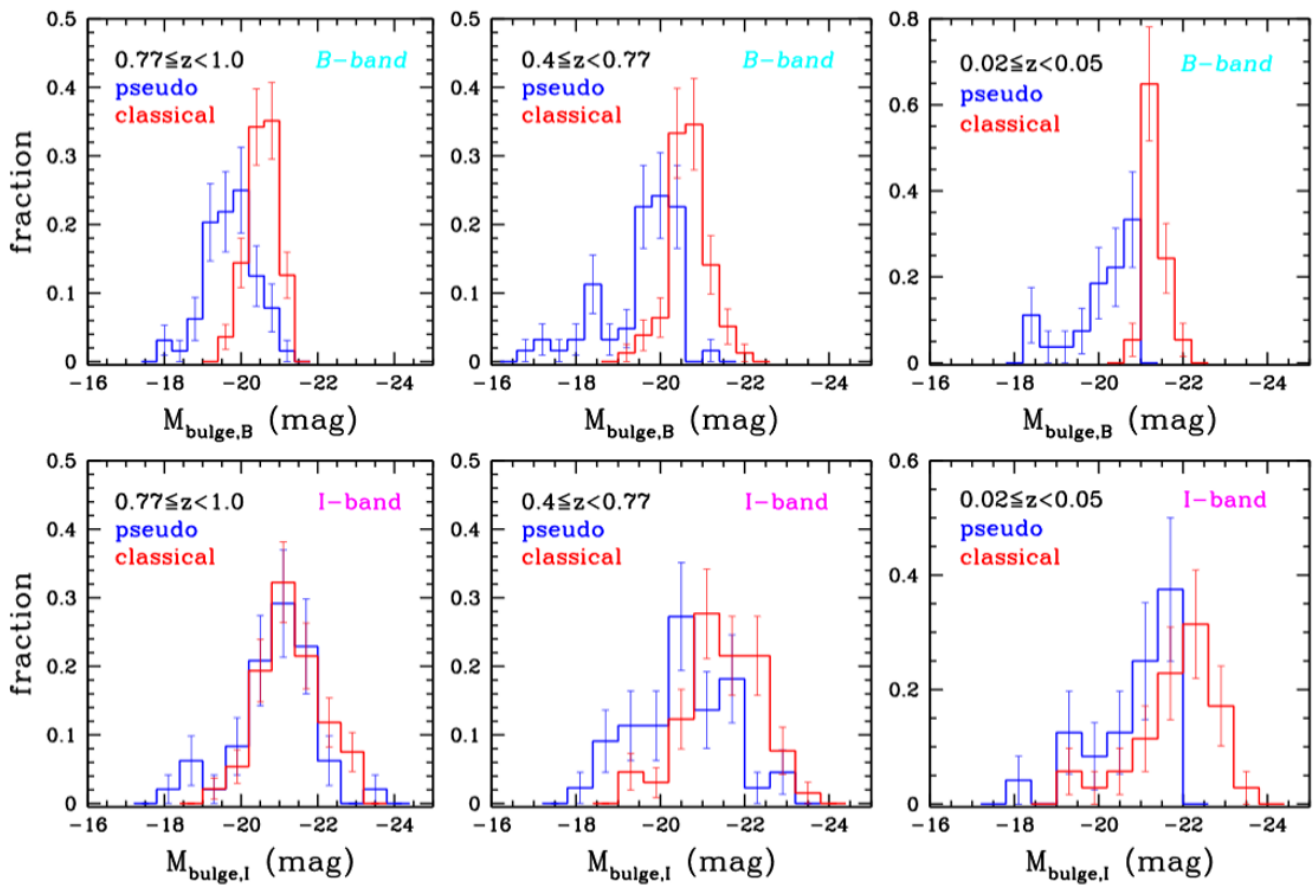

Figure 2. The distribution of bulge luminosity for pseudo bulges (blue) and for classical bulges (red), is shown for for three redshift ranges, in rest-frame B-band (top) and in rest-frame I-band, for HST Good-South (high $\mathrm{z}$ ) and SDSS $(\mathrm{z}=0)$ (Sachdeva et al. 2017 ).

classical ones, although the difference is fading at high $\mathrm{z}$ in the I-band (cf Figure 2). The frequency of bars decreases with z, as well as pseudo-bulges (Melvin et al. 2014). From a study on HST-COSMOS and SDSS or local galaxies, Kruk et al. $(2018)$ found that pseudo-bulges start to form about 7 Gyr ago, at $\mathrm{z}=0.7-0.8$.

Simulations have shown that the vertical rsonance in bars, responsible to the peanutshape of their bulges, can alco occur in nuclear bars. Is it possible to observe them? Peanut-shape bulges are easy to recognize in edge-on galaxies, however, if there is an embedded nuclear bar, it will be difficult to see it in projection on the larger bar. A nuclear bar is easier to distinguish in more face-on objects. However, it is possible to recognize a box-peanut bulge even face-on, through a barlens (Laurikainen et al. 2011; Laurikainen \& Salo 2017), or through the h4 parameter in its kinematics (Mendez-Abreu et al. 2008, 2014). The first box-peanut has been detected in a nuclear bar in NGC 1291 by Mendez-Abreu et al. (2019).

\section{Fueling and decoupling a secondary bar}

Simulations of the secular evolution of bars have shown how an embedded nuclear bar can decouple. First, the primary bar grows in amplitude by trapping more and more orbits, at larger radius, and with a lower precessing rate $(\Omega-\kappa / 2)$. This lowers the pattern speed of the bar $\Omega_{b}$, which soon develops two inner Lindblad resonances (ILR). Periodic orbits in between the two ILR are essentially the $\mathrm{x} 2$ family, which is perpendicular to the bar, and does not sustain the bar anymore. The bar is further weakened by the vertical thickening and the formation of the pseudo-bulge (Combes \& Sanders 1981). Then a new and faster bar develops inside the inner ILR ring (Friedli \& Martinet 1993 ). 

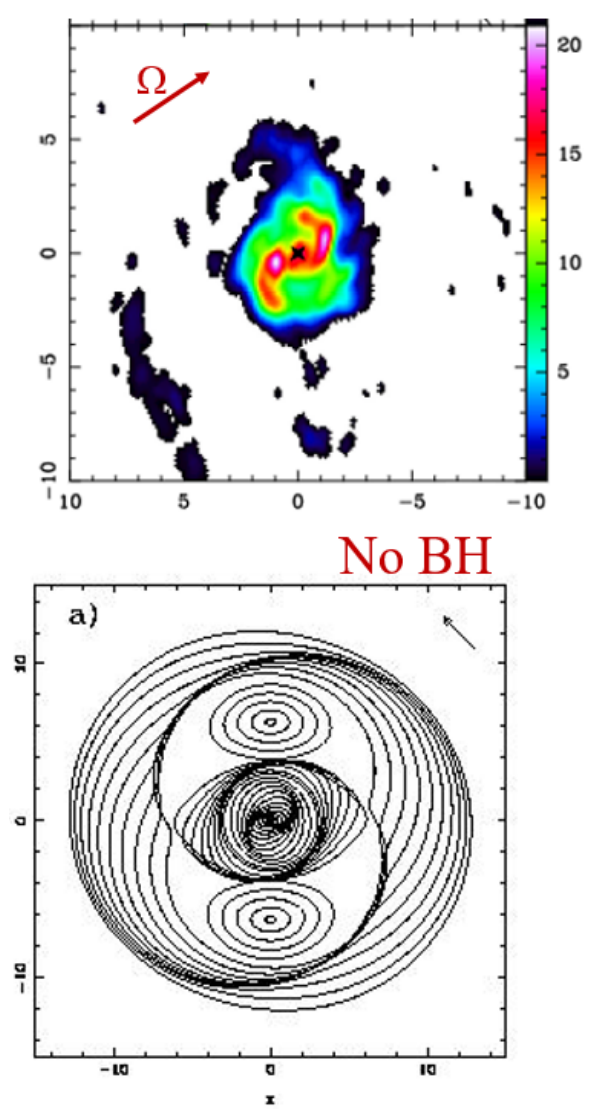

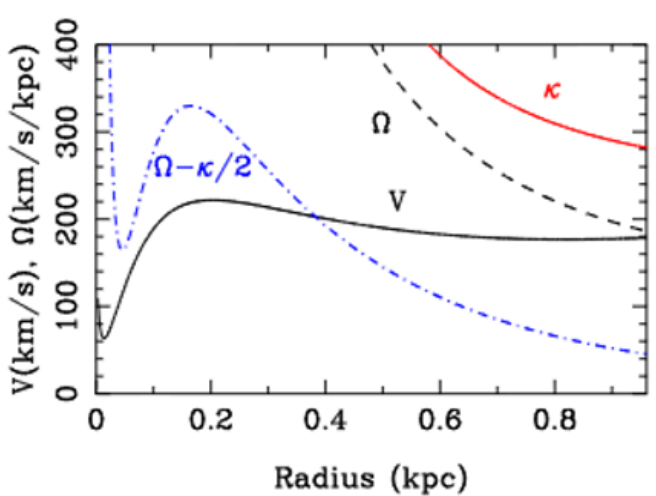

$\mathrm{BH}$

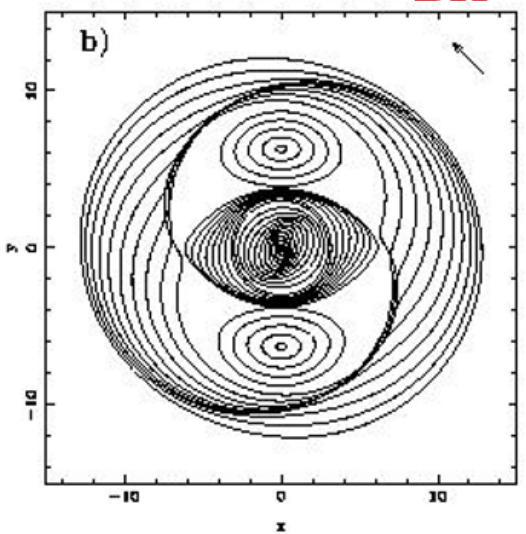

Figure 3. NGC1566 reveals a trailing nuclear spiral, of 70pc in radius (Combes et al. 2014): the de-projected map at the top left has been obtained in $\mathrm{CO}(3-2)$ with ALMA (axes in arcsec, $1=35 \mathrm{pc}$ ). The top right panel shows $\mathrm{V}_{\text {rot }}, \Omega, \kappa$ and $\Omega-\kappa / 2$, the precession rate of elliptical orbits. This rate rises again in the sphere of influence of the black hole, inside 50pc. Bottom: schematic gas streamlines in the bar rotating frame. The bar is horizontal. With no black hole, a leading spiral is expected, which becomes trailing with a black hole, when $\Omega-\kappa / 2$ rises again (Buta \& Combes 1996).

Since bars through their gravity torques drive gas towards the center, a cool nuclear disk accumulates, forming new stars with low velocity dispersion. This is at the origin of a drop in dispersion, frequently observed in the center of nearby Seyfert galaxies, and called $\sigma$-drop. It was observed through near infrared spectroscopy by Emsellem et al. (2001). The phenomenon was reproduced in simulations by Wozniak et al. (2003), taking into account the age of stellar populations, and their different mass-to-luminosity ratios. New simulations of $\sigma$-drop (Portaluri et al. 2017; di Matteo et al. 2019) have shown that the drop is detected in luminosity weighted lines until 10 Gyrs.

Gas driven inwards by the bars could fuel an active nucleus. However, AGN have duty cycles, and the fueling depends on the scales. In a previous study of the molecular gas in about 20 nearby Seyferts, with a spatial resolution of $\sim 100 \mathrm{pc}$, we conclude that only $\sim 35 \%$ of the objects revealed negative torques in the center Garcia-Burillo \& Combes 2012). The remaining two thirds of galaxies revealed positive torques, and gas was stalled in a ring at ILR. Since all galaxies should pass through the fueling phase, this means that the fueling phases are short, a few $10^{7} \mathrm{yrs}$, and are interrupted by feedback. Star 

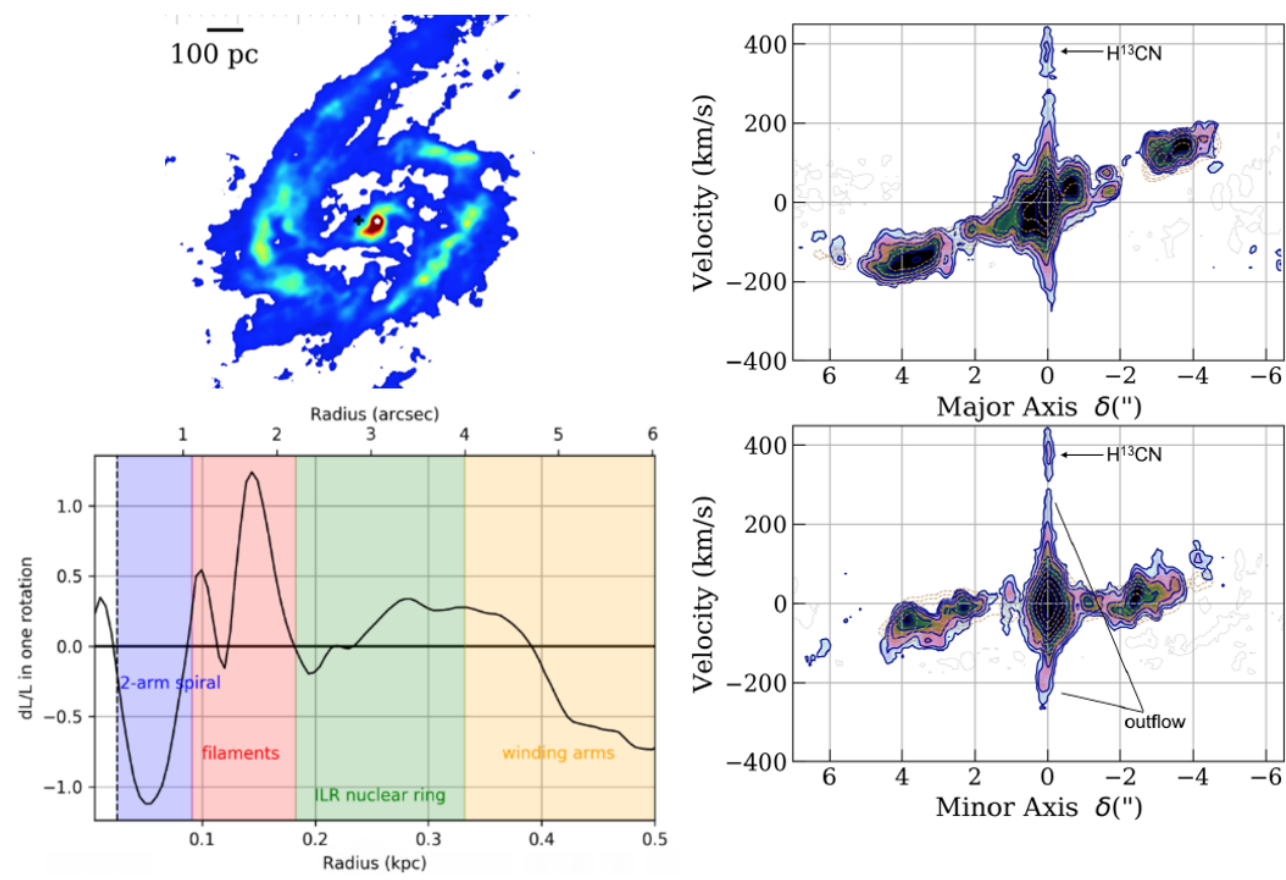

Figure 4. ALMA CO(3-2) map of the NGC 613 center, revealing a trailing nuclear spiral inside the ILR ring. At the bottom left, the gravity torques are negative inside 100pc, gas loses all its angular momentum in one rotation. At right, the PV diagrams on major and minor axes clearly show a molecular outflow (Audibert et al. 2019).

formation is also simultaneoulsy fueled by the torques, and is always associated to AGN activity, but can last during longer time-scales.

In the recent years, higher spatial resolution became available with ALMA. It then was possible to resolve the 10pc scale, and discover the fueling mechanism closer to the nucleus. For instance, in the case of NGC 1566 (cf Figure 3), in cycle 0 with a beam of $0.5 "=25 \mathrm{pc}$, we detected for the first time a nuclear trailing spiral of $\mathrm{R} \sim 70 \mathrm{pc}$ inside the ILR ring of radius $\sim 420 \mathrm{pc}$ (Combes et al. 2014). The trailing sense of the spiral was a surprise, since the gravity torques change sign at each resonance, and around the ILR ring, torques are negative outside and positive inside, so that gas accumulates in the ring. This is possible if, at least transiently, gas is in a leading spiral just inside the ring. But here the nuclear spiral enters in the sphere of influence of the black hole. The rotation velocity and associated frequencies displayed in Figure 3 show that the $\Omega-\kappa / 2$ curve is rising towards the center, and this explains the reversal of the winding sense of the spiral. This implies that torques are negative, and the nucleus is being fueled.

\section{Feedback, outflows, misalignment}

A nuclear trailing spiral has also been detected in NGC 1808 and NGC 613 (Combes et al. 2019, Audibert et al. 2019). Although a molecular outflow has been detected at larger scale in NGC1808 (Busch et al. 2017), there is no outflow in CO at small scale, close to the center, and we conclude that the outflow is rather due to the starburst feedback. The trailing nuclear spiral has a radius of $45 \mathrm{pc}$, and was observed with a beam of $0.08 "=4 \mathrm{pc}($ Audibert et al. 2019). The trailing spiral is also remarkable in the dense tracers maps: $\mathrm{HCN}(4-3), \mathrm{HCO}+(4-3), \mathrm{CS}(7-6)$. 
The case of NGC 613 is even more interesting, revealing both fueling due to a trailing nuclear spiral, but in simultaneity, a molecular outflow due to the AGN feedback (Audibert et al. 2019). With $0.09 \times 0.06$ resolution $(\sim 5 \mathrm{pc})$, the nuclear spiral is well resolved, with a radius of $60 \mathrm{pc}$, inside the ILR ring of $\mathrm{R}=300 \mathrm{pc}$. Inside the spiral, a torus, or circum-nuclar disk of radius $14 \mathrm{pc}$ is well decoupled and contrasted both in its morphology and kinematics, in particular in the dense tracers (Combes et al. 2019).

Figure 4 shows that the gas inside $100 \mathrm{pc}$ is losing quickly its angular momentum. The position-velocity diagrams along the major and minor axes reveal a molecular outflow (Audibert et al. 2019). The mass involved in the outflow is $210^{6} \mathrm{M}_{\odot}$, and the mass outflow rate is $27 \mathrm{M}_{\odot} / \mathrm{yr}$. This outflow could be entrained by a radio jet, but given the weak radio activity of NGC 613 , it might be a fossil outflow, reflecting its past activity.

Out of 8 galaxies observed at high resolution, we detected 7 molecular tori, or circumnuclear disks. Most of them have misaligned orientation and random inclinations with respect to the main spiral disk. This is not unexpected, given the very small scales sampled, and the very different time-scales involved. These tori are located within the sphere of influence of their black holes, and see an almost kepkerian spherical potential, with little influence of the outer disk. They might keep souvenir of their angular momentum, but the latter could be lost also through star formation feedback and associated fountain effect.

A high-resolution simulation of a Milky Way-like galaxy and the zoom in the central 200pc region, (Renaud et al. 2015, Emsellem et al. 2015) have shown how gas can fall back with any angular momentum. After a starburst episode in the center, a polar nuclear disk formed, which was rather long-lived, with respect to the dynamical time (Emsellem et al. 2015).

\section{Summary}

Classical bulges are supposed to form through interactions and mergers, while pseudobulges through internal evolution. The high frequency of pseudo-bulges or bulgeless galaxies among the local universe is a problem for the standard CDM model. It is possible to reduce the formation of spheroïds in a modified gravity model, where dynamical friction on dark matter halos is suppressed.

Bars through their gravity torques drive the gas from the galaxy disk towards the center, ad can fuel an active nucleus. Fueling phases are short however (a few $10^{7}$ yrs), and at a scale of $\sim 100 \mathrm{pc}$, only one third of nearby Seyfert are observed with negative torques. The decoupling of embedded nuclear bars or spirals may take over down to 10 pc scales.

With ALMA and improved resolution and sensitivity, it is now possible to resolve these scales, and several trailing nuclear spirals have been discovered, revealing the fueling of the AGN at small scales. Molecular tori or circum-nuclear disks of 10-20 pc in sizes have been detected, randomly oriented with respect to the main galaxy disk. In some galaxies, molecular outflows are also observed, either due to the supernovae feedback or to a radio jet. The coupling of the AGN feedback is enhanced by the random orientation of the jets. The misalignment between small and large scales are not unexpected, especially inside the sphere of influence of the black hole. The gas may lose its angular momentum orientation, due to stellar feedback and the fountain effect.

\section{References}

Audibert, A., Combes, F., Garcia-Burillo, S. et al. 2019, AEAA, in press., arXiv1905.01979 
Busch, G., Eckart, A., Valencia-S, M. et al. 2017, A $₫ A$, 598, A55

Buta, R., \& Combes, F. 1996, Fund. Cosmic Phys., 17, 95

Ceverino, D., Dekel, A., Bournaud, F. 2010, MNRAS, 404, 2151

Combes, F., Sanders, R.H 1981, A\&A, 96, 164

Combes, F., Garcia-Burillo, S., Casasola, V. et al. 2014, A\&A, 565, A97

Combes, F. 2014, A\&4A, 571, A82

Combes, F., Garcia-Burillo, S., Audibert, A et al. 2019, AधA, 623, A79

Di Matteo, P., Fragkoudi, F., Khoperskov, S. et al. 2019, A\& A, 628,A11

Emsellem, E., Greusard, D., Combes, F. et al. 2001, A\&A, 368, 52

Emsellem, E., Renaud, F., Bournaud, F. et al. 2015, MNRAS, 446, 2468

Fisher, D. B., Drory, N. 2010, Ap. J., 716, 942

Garcia-Burillo, S., Combes, F. 2012, JPhCS, 372, a2050

Friedli, D., Martinet, L. 1993, A\&A, 277, 27

Kormendy, J., Fisher, D.B. 2008, ASPC, 396, 297

Kruk, S. J., Lintott, C. J., Bamford, S. P. et al. 2018, MNRAS, 473, 4731

Laurikainen, E., Salo, H. 2017, A $6 A$, 598, A10

Laurikainen, E., Salo, H., Buta, R., Knapen, J. H. 2011, MNRAS, 418, 1452

Melvin, T., Masters, K., Lintott, C., et al. 2014, MNRAS, 438, 2882

Mendez-Abreu, J., Corsini, E. M., Debattista, V. P. et al. 2008, Ap. J., 679, L73

Mendez-Abreu, J., Debattista, V. P., Corsini, E. M., Aguerri, J. A. L. 2014, A\& A, 572, A25

Mendez-Abreu, J., de Lorenzo-Caceres, A., Gadotti, D. A. et al. 2019, MNRAS, 482, L118

Mishra, P. K., Wadadekar, Y., Barway, S. 2017, MNRAS, 467, 2384

Portaluri, E., Debattista, V. P., Fabricius, M. et al. 2017, MNRAS, 467, 1008

Renaud, F., Bournaud, F., Emsellem, E. et al. 2015, MNRAS, 454, 3299

Sachdeva, S., Saha, K., Singh, H. P. 2017, Ap. J., 840, 79

Wang, L., Wang, L., Li, C. et al. 2019, MNRAS, 484, 3865

Weinzirl, T., Jogee, S., Khochfar, S. et al. 2009, Ap. J., 696, 411

Wozniak, H., Combes, F., Emsellem, E., Friedli, D. 2003, A\&A, 409, 469 